\section{Failure to sterilize in plastic bags}

\author{
P. H. EVERALL and C. A. MORRIS Public Health \\ Laboratory and Department of Microbiology, Mytton \\ Oak Road, Shrewsbury
}

The ideal container for discarded cultures and other contaminated materials which require autoclaving to render them safe should be reasonably cheap, resistant to corrosion, leak-proof, and capable of allowing the passage of steam to all parts of its contents. Perhaps because of the difficulty of meeting all these requirements with the metal containers which have traditionally been used some laboratories have changed in recent years to plastic bags. Such bags are sold as being suitable for the sterilization of discarded plastic Petri dishes and other containers, with the recommendation that they should be only loosely tied. One supplier will also provide metal ties for closure.

In the course of routine surveillance of laboratory autoclave loads it was noted that plastic bags frequently performed very badly, as judged by thermocouple measurements. Accordingly, tests were carried out to investigate the problem further, as a result of which we present evidence that all-plastic bags (as distinct from composite paper and plastic bags) are often unsuitable because of the slow penetration of steam into them.

In the first instance we performed experiments to compare all-plastic bags with metal containers, employing as far as possible comparable loads of glass or plastic in each. The bags invariably performed worse than did the metal containers, and this was true irrespective of whether the autoclave was a pure downwards-displacement instrument or a vacuum-assisted one. However, it was difficult to obtain reproducible results from the bags as these tended to soften and deform when heated. Furthermore, it was not possible to draw firm conclusions from such experiments owing to variations in the size and contents of the containers. Accordingly, in later experiments, an artificial load consisting of polypropylene universal container caps was used. Bags of the same size, composed of either allplastic or plastic and paper, were compared with each other and with traditional metal containers. Autoclaving was carried out at $126^{\circ} \mathrm{C}$ (measured

Received for publication 21 July 1976

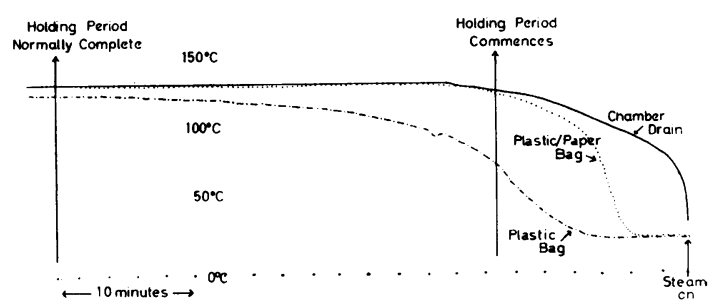

Figure Temperatures recorded by thermocouples in the autoclave chamber drain, in a composite plastic and paper bag, and in an all-plastic bag. Both the latter contained polypropylene universal container caps. A temperature of $126^{\circ} \mathrm{C}$ was achieved for 28 minutes in the paper and plastic bag, whereas at the end of the holding period the temperature in the all-plastic bag was only $123^{\circ} \mathrm{C}$.

from the chamber drain) and this temperature was maintained for 30 minutes. Under these conditions loads in our metal containers regularly reach $126^{\circ} \mathrm{C}$ for a minimum of 10 minutes. However, even small all-plastic bags (dimensions $40 \times 25 \mathrm{~cm}$ ) did not attain sterilizing temperature (figure) and failed to do so even when the holding period was proß longed to one hour. Bags of the same size constructed of plastic and paper performed excellently, the loads reaching sterilizing temperature almost simultaneously with the chamber drain. Thus it was demonstrated that the failure to achieve sterilizing temperature within the bag was not an intrinsic property of the load but was peculiar to the all-plastic bag because of inadequate removal of air.

It is of interest that a number of central sterile supply departments which at one time used plastic bags of nylon, have abandoned their use because of poor steam penetration, replacing them with the composite type of bags which, by our tests, were found to perform well.

We recommend that all-plastic bags should be used with great caution. Where they are used for the sterilization of infected materials steps should be taken to establish that a sterilizing time/temperature relationship is in fact being obtained. Prolongation of the holding period may be no solution for if steam penetration should be completely prevented the process may become one of dry heat sterilization with consequently very different requirements for temperatures and times. 